\title{
The spontaneous motion of a slug of miscible liquids in a capillary tube
}

\author{
Mathieu Sellier* \\ Department of Mechanical Engineering, \\ University of Canterbury, \\ Private Bag, 4800, \\ Christchurch, 8140, New Zealand \\ Fax: +64 33642078 \\ Email: mathieu.sellier@canterbury.ac.nz \\ ${ }^{*}$ Corresponding author
}

\section{Claude Verdier}

Laboratoire Interdisciplinaire de Physique (LIPhy), CNRS and Université Grenoble Alpes (UMR 5588), F-38000, France

Email: claude.verdier@ujf-grenoble.fr

\section{Volker Nock}

Department of Electrical and Computer Engineering, University of Canterbury,

Private Bag, 4800,

Christchurch, 8140, New Zealand

Fax: +64 33642078

Email: volker.nock@canterbury.ac.nz

\begin{abstract}
This contribution explores a droplet actuation mechanism which involves mixing slugs of two different liquids in a glass capillary. The resulting contrast in surface tension which arises provides the necessary propulsive power for the droplet. The conceptual idea is demonstrated for an ethanol-water system. The droplet is observed to rapidly reach a peak velocity which then gradually decreases with time as the two miscible liquids mix. A model is proposed based on Newton's second law which is able to capture the main observed flow phenomena and explain the driving and dissipative mechanisms simultaneously at play in the droplet. This passive actuation mechanism could prove an attractive alternative in digital microfluidics systems for which bulky pumping systems are often required.
\end{abstract}

Keywords: droplet; self-propulsion; microfluidics; multiphase flow; miscible liquids; contact line; capillary; wetting.

Reference to this paper should be made as follows: Sellier, M., Verdier, C. and Nock, V. (2017) 'The spontaneous motion of a slug of miscible liquids in a capillary tube', Int. J. Nanotechnol., Vol. 14, Nos. 1/2/3/4/5/6, pp.530-539. 
Biographical notes: Mathieu Sellier is an Associate Professor in Mechanical Engineering at the University of Canterbury. His research interests span a wide range related to the modelling of fluid flow phenomena. Prior to his appointment at the University of Canterbury in 2006, he was a Postdoctoral at the Fraunhofer-ITWM institute in Kaiserlautern, Germany, developing numerical tools for the modelling and optimisation of glass forming technologies. He got his PhD from the University of Leeds, UK, in 2003 for a thesis on the modelling of thin liquid films and droplets on complex surfaces.

Claude Verdier is Research Director at CNRS in the Laboratory of Interdisciplinary Physics (LIPhy, Grenoble). He received an Engineering degree at Ecole Centrale Paris (France) in 1986 and a PhD in Mechanics at the University of Minnesota in 1990. He currently supervises research in complex fluids and biorheology, in particular problems relevant to cancer metastasis. He is currently in charge of a sector at CNRS, heads a French Research Group on 'biomechanics' and is a PI of a project on 'cancer cell transmigration'.

Volker Nock is a Senior Lecturer in the Department of Electrical and Computer Engineering at the University of Canterbury. He received his Dipl.-Ing. in Microsystem Technology from the University of Freiburg, Germany, in 2005 and his PhD from the University of Canterbury, New Zealand, in 2009. Before becoming a lecturer, he held both a MacDiarmid Institute and a Marsden Fund Postdoctoral Fellowship. He is currently a Principal Investigator of the Biomolecular Interaction Centre and an Associate Investigator of the MacDiarmid Institute for Advanced Materials and Nanotechnology.

\section{Introduction}

With the increasing demand of miniaturisation of medical devices and increased throughput, the concept of lab-on-a-chip emerged and grew over the past two decades. In this context, the idea that liquid droplets could be used effectively as test tubes by confining within them analytes and reagents arose and digital microfluidics was born. Droplets can be transported in microchannels, reactions induced by mixing the reacting droplets, and with appropriate sensors the result of these reactions can be monitored and analysed. One of the drawbacks of such microfluidic systems is that they often require bulky pumping systems to circulate the fluids in the device. In the spirit of the work of Beebe and co-workers [1,2], we explore in this paper whether the unique flow phenomena which occur at the small scale of microfluidic devices may be exploited to spontaneously actuate droplets confined in a microchannels. We will refer to such droplets as slug in the following. The driving force we propose to exploit is the gradient of surface energy which arises when two droplets of different miscible liquids are placed side by side and led to coalesce in a capillary tube. Because of the asymmetry of the system with one side of the droplet mostly composed of one fluid and the other side mostly composed of the other fluid, one expects that if the surface tension difference is large enough, it will provide a driving force able to overcome viscous resistance and induce the motion of the slug down the tube. In principle, one expects this motion to be sustained until both fluids have fully mixed and hence surface energy has homogenised. The present authors have already demonstrated this actuation principle in an unconfined 
system, i.e., when the droplets are exposed to the surrounding atmosphere [3,4]. In this paper, we demonstrate that slugs can successfully be propelled in a capillary tube by mixing two different miscible liquids. We also propose a simple phenomenological model which explains some of the observed phenomena and expected scaling laws in terms of the key parameters of the problem such as the capillary diameter or the liquid properties. In the early 2000, Bico and Quére [5,6] studied the closely related problem of self-propulsion of slugs of immiscible liquids. In this work, the spontaneous motion is also observed, but a thin layer of one of the liquid phases remains attached to the wall of the capillary tube effectively creating a coating layer. The motion of the system for immiscible liquid is sustained until the liquid from the slug, which is transferred into the coated layer on the wall of the capillary tube, is exhausted. Bico and Quéré $[5,6]$ also alluded to the case of miscible liquid, but did not explore it to great depth. We build on this work here and present experimental data and a corresponding mathematical model.

The paper is organised as follows. The next section describes briefly how the experiment was set-up and present illustrative results. Section 3 describes the mathematical model which describes the main flow characteristics. In Section 4, the experimental and numerical models are compared and scaling laws expected to govern the slug motion are presented. Finally, the paper closes with Section 5.

\section{Experimental results}

A small plug of dyed distilled water was first drawn into the glass capillary by simply dipping it into a container of dyed distilled water. The liquid rose in the tube by capillary suction. The amount of liquid drawn into the tube can be controlled by the time it is immersed in the container (provided the final equilibrium height of liquid in the capillary tube has not been reached). The radius of the capillary tube which was used is $0.675 \mathrm{~mm}$. The capillary tube was then placed horizontally on a white paper sheet for maximum imaging contrast close to a graduated ruler with the plug of dyed distilled water at one extremity of the tube. A plug of ethanol was then gently inserted using a micropipette at the extremity of the capillary tube where the water plug is present. Care was taken to transfer minimum momentum during this insertion. The experiment can be viewed on the video available as supplementary material or at http://youtu.be/KVr2dtvvAGU. A couple of still frame images can be seen in Figure 1.

As coalescence occurs, the slug is seen to move down the capillary tube in the absence of any apparent applied force. The motion is, as expected, in the direction of the liquid of higher surface tension, i.e., the dyed water in this case, and therefore away from the extremity of the capillary tube. The motion is sustained until both liquids have fully mixed. Therefore, it is expected that the slug velocity will be maximum early on and decay towards zero as time progresses. This is confirmed by Figure 2, which shows the velocity of the slug as a function of time. The maximum velocity observed is on the order of a few $\mathrm{mm} / \mathrm{s}$. The intensity of the blue dye (most clearly seen on the video) is also a good indicator of the mixing rate of the two liquids. 
Figure 1 Slug evolution in the glass capillary. As time progresses, the slug moves from left to right (see online version for colours)
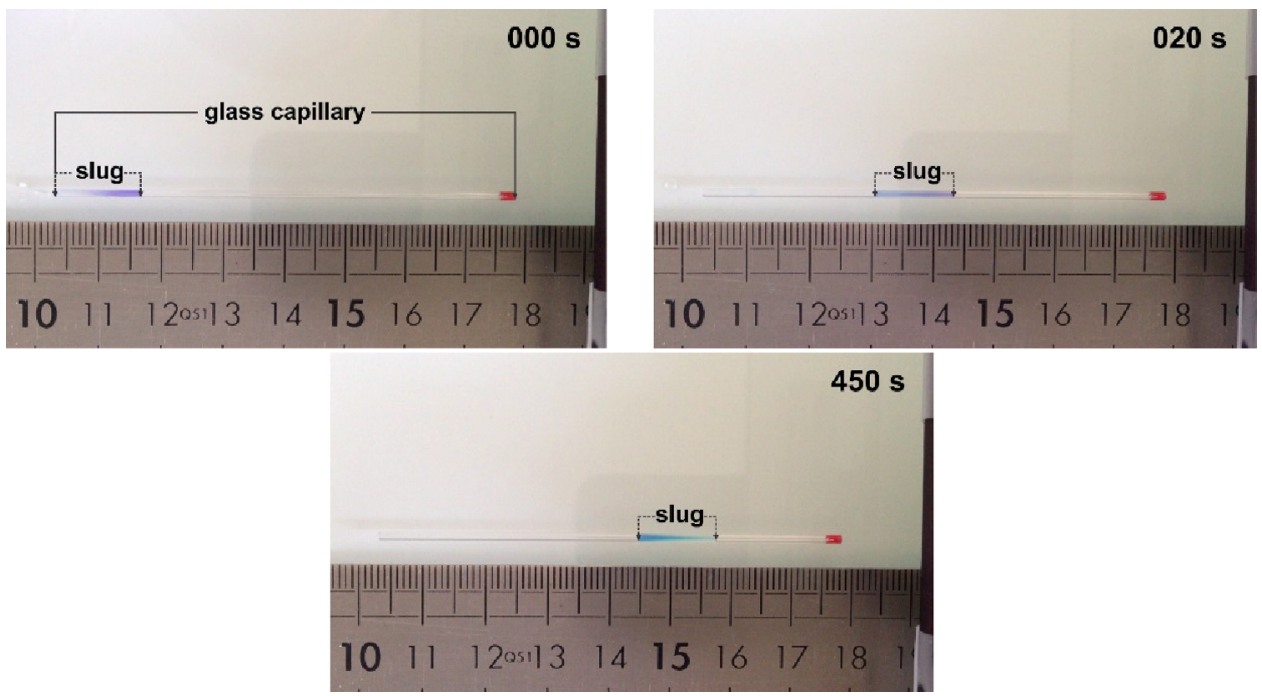

Figure 2 Position of the edges of the slug as a function of time (a) and velocity of the slug as a function of time (b) (see online version for colours)

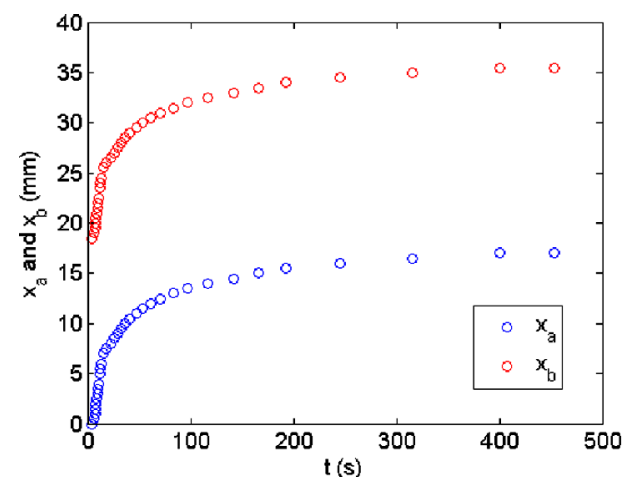

(a)

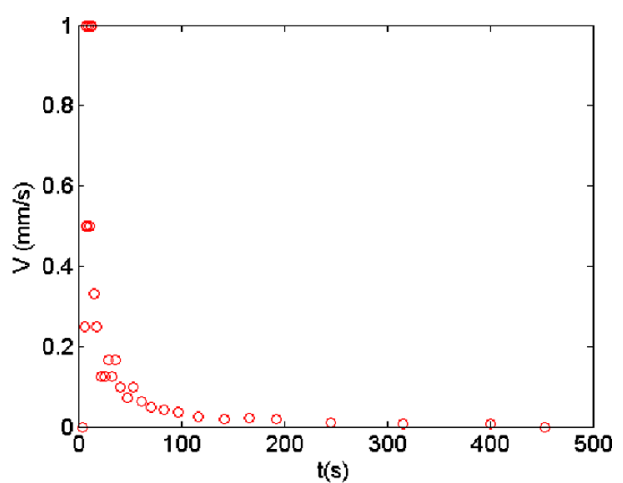

(b)

\section{Phenomenological model}

With the above phenomena observed, there remains to explain the main flow features through a basic fluid mechanics analysis which is adapted from the work of Bico and Quéré [6]. The basis of this analysis is Newton's second law applied to the slug shown in Figure 3 . In this sketch, the slug moves from left to right with a velocity $V(t), D_{1}$ indicates the volume initially occupied by ethanol and $D_{2}$ that initially occupied by the water. Referring to Figure 3, the forces which apply on the liquid slug are:

- A net surface tension force applied on the perimeter of the capillary tube of radius $R$, $2 \pi R\left(\gamma_{r} \cos \left(\theta_{a}\right)-\gamma_{l} \cos \left(\theta_{r}\right)\right)$, where $\gamma_{r}, \gamma_{l}$ are the surface tensions on the right-hand 
and left-hand sides of the slugs and $\theta_{a}, \theta_{r}$ are the advancing and receding contact angles, respectively.

- A viscous force which results from the shear stress developed at the wall of the capillary tube. This force can be expressed as $8 V\left(\mu_{w} l_{w}+\mu_{e} l_{e}\right) \pi$ where $\mu_{w}, \mu_{e}$ are the dynamic viscosities of pure water and pure ethanol and $l_{w}, l_{e}$ are the initial length of the water and ethanol slugs.

- A net viscous dissipation term at the moving contact which has been shown in De Gennes [7] to be equal to

$$
2 \pi R\left(\frac{3 \mu_{e} V \Gamma}{\theta_{r}}+\frac{3 \mu_{w} V \Gamma}{\theta_{a}}\right) \text {. }
$$

This dissipation force is evaluated by calculating the viscous stress in a wedge which moves with the velocity of the advancing or receding contact line. Balancing the non-compensated capillary force with the viscous force close to the contact line leads to

$$
\theta_{d}=\left(\frac{6 \Gamma \mu V}{\gamma}\right)^{1 / 3}
$$

where $\theta_{d}$ is the dynamic contact angle (either advancing or receding) and $\Gamma$ involves the natural logarithm of the macroscopic and molecular length scales. It is thought to have a value close to 15 [6] which is the value adopted in the following.

Figure 3 Sketch of the slug and the corresponding force balance (see online version for colours)

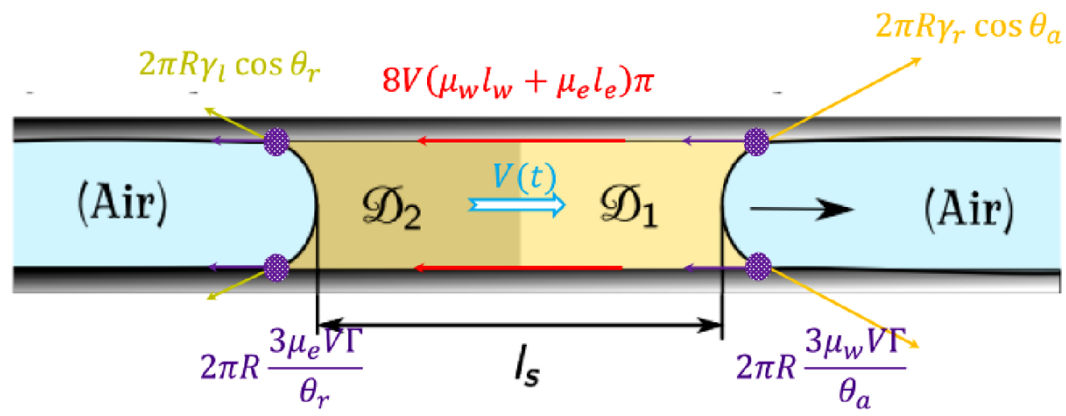

Hence, from Newton's second law, we can deduce

$$
\begin{aligned}
m \frac{\mathrm{d} V}{\mathrm{~d} t}= & 2 \pi R\left(\gamma_{r} \cos \left(\theta_{a}\right)-\gamma_{l} \cos \left(\theta_{r}\right)\right)-8 V\left(\mu_{w} l_{w}+\mu_{e} l_{e}\right) \pi \\
& -2 \pi R\left(\frac{3 \mu_{e} V \Gamma}{\theta_{r}}+\frac{3 \mu_{w} V \Gamma}{\theta_{a}}\right)
\end{aligned}
$$


The surface tensions $\gamma_{r}, \gamma_{l}$ are functions of the mixture composition. The surface tension of pure ethanol $\gamma_{e}$ is $0.022 \mathrm{~Pa} . \mathrm{m}$ and that of pure water $\gamma_{w}$ is $0.71 \mathrm{~Pa} . \mathrm{m}$. For water-ethanol mixtures, the corresponding surface tension has been measured in Vazquez et al. [8] and is well described by the following correlation:

$$
\frac{\gamma_{w}-\gamma(c)}{\gamma_{w}-\gamma_{e}}=\frac{1+a(1-c)}{1-b(1-c)} c
$$

where $(a, b)=(0.917,0.956)$ are constants and $c$ is the concentration of ethanol in the mixture. To proceed further, we need a model which describes the concentration of ethanol in the moving slug. Two mechanisms are responsible for the mixing of ethanol and water. The first one is molecular diffusion. The coefficient of diffusion for liquid ethanol in liquid water is $D=1.6 \times 10^{-9} \mathrm{~m}^{2} / \mathrm{s}$ [9]. An additional transport mechanism which is responsible for the mixing of ethanol and water is convection. It was shown by Taylor in the 1950s [10] that in a reference frame which moves with the slug, mixing can be represented by an effective diffusion coefficient $D_{\text {eff }}$, which is the sum of the molecular diffusion $D$ and Taylor dispersion such that

$$
D_{\text {eff }}=D+\frac{R^{2} V^{2}}{48 D}
$$

Accordingly, in a reference frame which moves with the slug, the concentration of ethanol must satisfy the following equation:

$$
\frac{\partial c}{\partial t}=\frac{\partial}{\partial x}\left(D_{\text {eff }} \frac{\partial c}{\partial x}\right)
$$

subject to the following initial and boundary conditions

$$
c(x, t=0)=1-H\left(x=l_{1}\right) ;\left.\frac{\partial c}{\partial x}\right|_{x=0}=\left.\frac{\partial c}{\partial x}\right|_{x=L}=0
$$

where $H$ is the Heaviside function and $L$ the total length of the slug. The zero slope boundary condition is justified by the fact that we expect no ethanol flux at either end of the slug. There exists an analytical solution to the above diffusion problem with the following form:

$$
c(x, t)=\frac{l_{1}}{L}+\sum_{n=1}^{\infty} \frac{2}{n \pi} \sin \left(\frac{n \pi l_{1}}{L}\right) \cos \left(\frac{n \pi x}{L}\right) \exp \left(-D_{\text {eff }}\left(\frac{n \pi}{L}\right)^{2} t^{2}\right)
$$

Figure 4 illustrates the ethanol concentration variation and the corresponding variation in surface tension for a hypothetical value of the diffusion constant $D_{\text {eff }}=3 \times 10^{-6} \mathrm{~m}^{2} / \mathrm{s}$ and $L=1.8 \mathrm{~cm}, l_{1}=1 \mathrm{~mm}$. These parameter values are representative of actual values reported in the next section.

The ordinary differential equation, equation (1), resulting from the mathematical model is easily solved in MATLAB using the ode 45 routine. 
Figure 4 Variation of ethanol concentration as a function of space and time (a); Corresponding variation of surface tension at the water and ethanol sides (b) (see online version for colours)

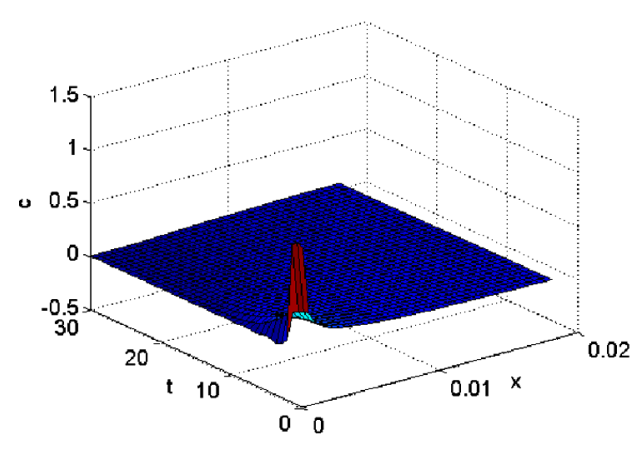

(a)

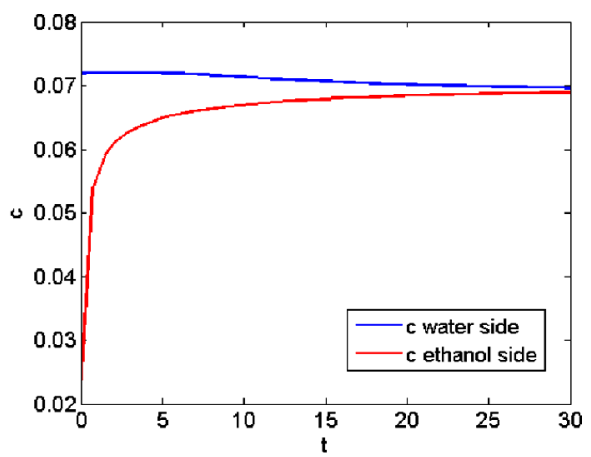

(b)

\section{Results and scaling laws}

To assess the validity of the model developed above, we calculate the velocity predicted using equation (1) for the experimental run shown in Figure 2 and compare with the measured velocity. In that case, the slug length $L$ is $1.8 \mathrm{~cm}$, the initial ethanol slug length $l_{1}$ is $1 \mathrm{~mm}$, the capillary tube radius is $0.675 \mathrm{~mm}$, the viscosity of pure water is $1 \times 10^{-3} \mathrm{~Pa}$.s and that of ethanol $1.261 \times 10^{-3} \mathrm{~Pa}$.s. The results are summarised on Figure 5, confirming that the model is able to predict the magnitude and trend of the velocity variation reasonably well.

Figure 5 Comparison between the velocity predicted by the model (in $\mathrm{m} / \mathrm{s}$ ) and that measured experimentally (see online version for colours)

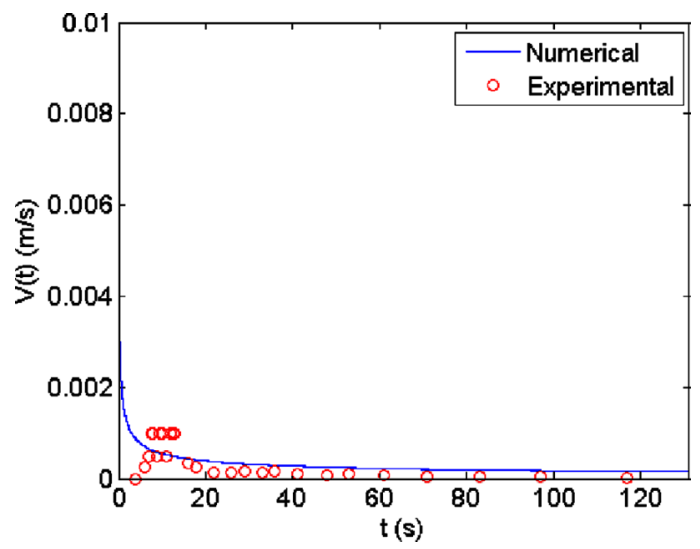

Unfortunately, we could not capture experimentally the early dynamic to truly validate the model at short times. Nonetheless, a number of things can be stated: the model correctly predicts a peak initial velocity which decays as time proceeds as observed in experiments. Taylor dispersion plays a key role in the mixing mechanism. Molecular diffusion alone cannot possibly account for the velocity decay rate. Numerical 
experiments with the molecular diffusion alone shows that the decay rate would be much slower (data not shown).

With increased confidence that the proposed model is relevant to describe the slug dynamics, we next concentrate on trying to deduce scaling laws governing the total distance travelled by the slug. We expect the travelled distance $L_{t}$ to scale like the product of the maximum velocity, $V_{\max }$, with the time required for ethanol and water to fully mix $\left(T_{d}\right)$ since motion will cease beyond that time. The time required for mixing should be:

$$
T_{d} \sim L^{2} / D_{\text {eff }}=L^{2} / D\left(1+\frac{R^{2} V_{\max }^{2}}{48 D^{2}}\right)
$$

If we assume that the maximum velocity is given by balancing the net surface tension driving force (the first force in the list of forces on the slug reported above) with the bulk viscous dissipation (the second force in that list), we obtain that

$$
V_{\max } \sim \frac{R \Delta(\gamma \cos (\theta))}{4 \mu L}
$$

Combining equations (7) and (8) and assuming that Taylor dispersion dominates over molecular diffusion leads to the following scaling law

$$
\frac{L_{t}}{L} \sim \frac{L D}{R^{2} V_{\max }}
$$

Accordingly, the slug travelled distance should increase linearly with the slug length and the diffusion coefficient but should decrease with the square of the capillary radius. This scaling law is tested numerically by systematically varying $L, D$ and $R$ from the base case described previously and computing the travelled distance using the theoretical model. Note that the total length of the slug is varied but the initial ethanol concentration is kept the same, i.e., $l_{1} / L$ is constant. The results, shown in Figure 6 , confirm that the travelled distance varies linearly with the slug length, the diffusion coefficient, and the inverse of the capillary radius squared since the numerical results are perfectly fitted with lines.

The fact that this scaling law holds allows us to make an additional inference. Namely, $V_{\max }$ in equation (9) cannot possibly be a function of $L, D$ and $R$ as if it were, different exponents would arise for $L, D$ and $R$ in the scaling law (equation (9)). This implies that the maximum velocity is not dictated by an initial balance between the driving capillary pressure and the viscous dissipation in the bulk (terms 1 and 2 in the list of forces on the slug). If it were, the maximum velocity would be given by equation (8) which is a function of $L$ and $R$ hence invalidating the scaling law. The only other possibility is that the maximum velocity is dictated by a balance between the driving capillary pressure and the viscous dissipation at the contact line (terms 1 and 3 in the list of forces on the slug). In other words, at the early stages, the model suggests that most of the energy dissipated by viscous stresses occurs in a region close to the contact lines instead of in the bulk of the slug. And this balance between surface tension driving force and viscous dissipation at the contact line dictates the magnitude of the maximum velocity. 
Figure 6 Effect of $R, L$ and $D$ on the slug travelled distance. The symbols indicate the results from the simulations and the full lines linear fits (see online version for colours)
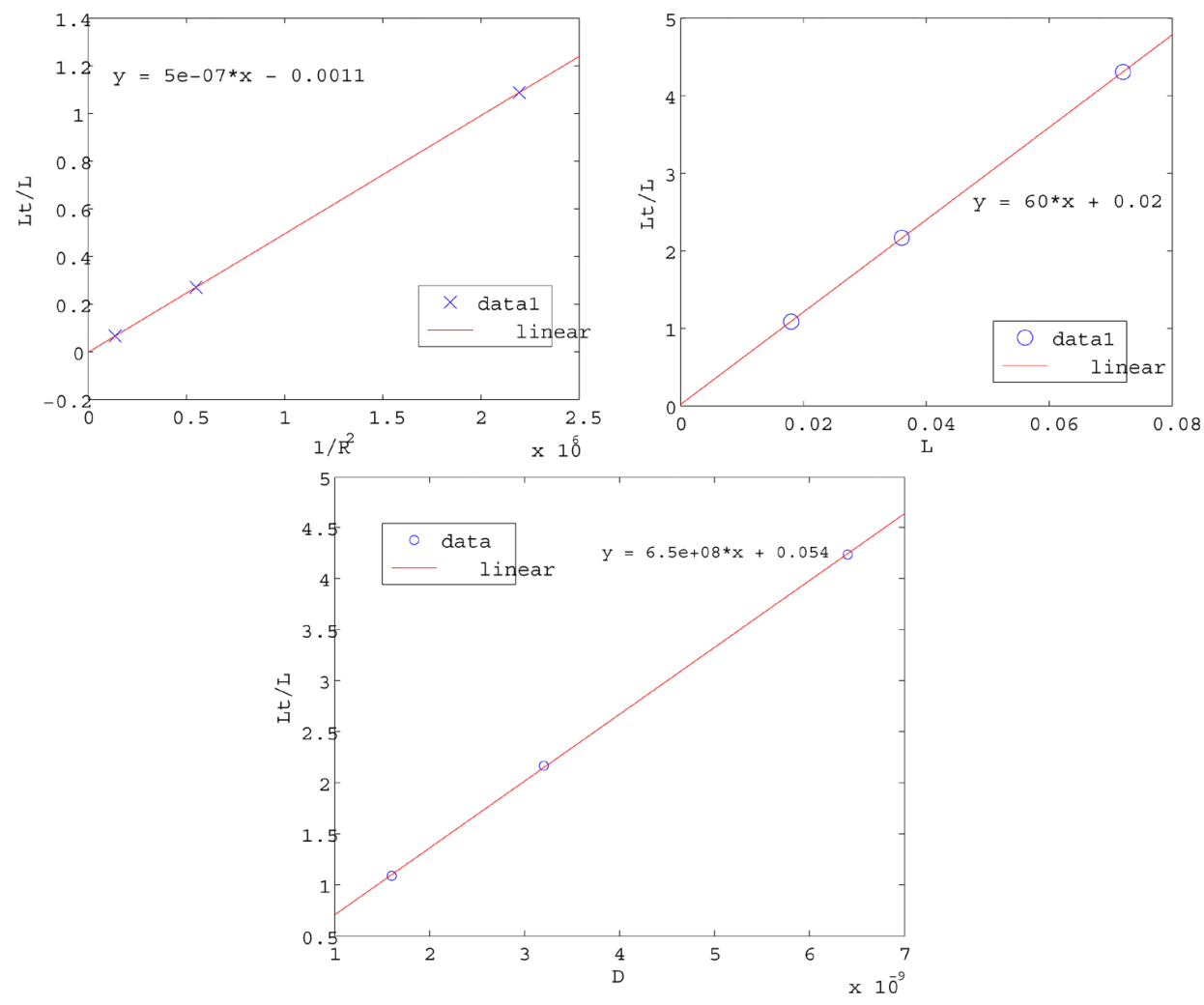

\section{Concluding remarks}

In this paper, we have demonstrated that the surface energy imbalance which arises at the extremities of a slug of miscible liquids is able to induce the spontaneous motion of the slug confined in a capillary tube. To demonstrate this phenomena, we considered a water-ethanol system and the results are illustrated in the video available at http://youtu.be/KVr2dtvvAGU. The slug clearly moved in the direction of initial water slug having the higher surface tension. The motion was sustained for a while but the velocity of the systems gradually decreased. This is attributed to the mixing of the two liquids, which results in the balance of surface energies on either side. Besides demonstrating the feasibility of this actuation mechanism, we have also proposed a simple model able to capture the main features of the slug motion. This model is based on Newton's second law and predicts the initial peak velocity followed by a gradual velocity decay. Forces which are accounted for include net surface tension force, viscous dissipation at the wall in the slug bulk and net viscous dissipation at the contact line region. Our results confirm the importance of Taylor dispersion in the mixing of the two phases. Taylor dispersion, effectively representing mixing by convection, increases the mixing rate by several orders of magnitude and hence shortens the travelling time of the slug since motion ceases when the slug is fully mixed. Using simple scaling arguments, 
we were also able to show that the slug travelled distance is expected to increase linearly with slug length, diffusion coefficient, and the inverse of the squared capillary radius. Furthermore, this result suggests that the main mechanisms for energy dissipation are viscous stresses at the slug contact lines (advancing or receding).

\section{Acknowledgements}

This work was supported by the Royal Society of New Zealand through the Marsden grant (Grant No. UOC1104). Useful discussions with David Quéré and Irshad Khodabocus are also gratefully acknowledged.

\section{References}

1 Atencia, J. and Beebe, D.J. (2005) 'Controlled microfluidic interfaces', Nature, Vol. 437, pp.648-655.

2 Berthier, E. and Beebe, D.J. (2007) 'Flow rate analysis of a surface tension driven passive micropump', Lab Chip, Vol. 7, No. 11, pp.1475-1478.

3 Sellier, M., Nock, V. and Verdier, C. (2011) 'Self-propelling, coalescing droplets', Int. J. Multiphase Flow, Vol. 37, No. 2, pp.462-468.

4 Sellier, M., Nock, V., Gaubert, C. and Verdier, C. (2013) 'Droplet actuation induced by coalescence: experimental evidences and phenomenological modeling', Eur. Phys. J. Spec. Top., Vol. 219, No. 1, pp.131-141.

5 Bico, J. and Quéré, D. (2000) 'Liquid trains in a tube', Europhys. Lett., Vol. 51, p.546.

6 Bico, J. and Quéré, D. (2003) 'Self-propelling slugs in a tube', J. Fluid Mech., Vol. 467, p.201.

7 De Gennes, P.G. (1985) 'Wetting: statics and dynamics', Rev. Mod. Phys., Vol. 57, p.827.

8 Vazquez, G., Alvarez, E. and Navaza, J.M. (1995) 'Surface tension of alcohol water+ water from 20 to 50 degree. C', J. Chem. Eng. Data, Vol. 40, No. 3, pp.611-614.

9 Hills, E.E., Abraham, M.H., Hersey, A. and Bevan, C.D. (2011)'Diffusion coefficients in ethanol and in water at 298K: linear free energy relationships', Fluid Phase Equilib., Vol. 303, No. 1, pp.45-55.

10 Taylor, G.I. (1953) 'Dispersion of soluble matter in solvent flowing slowly through a tube', Proc. R. Soc. London, A, Vol. 219, No. 1137, pp.186-203. 